\title{
Difficidin and bacilysin produced by plant-associated Bacillus amyloliquefaciens are efficient in controlling fire blight disease
}

\author{
X.H. Chen ${ }^{a}$, R. Scholz ${ }^{a}$, M. Borriss ${ }^{b}$, H. Junge ${ }^{b}$, G. Mögel ${ }^{c, d}$, S. Kunz ${ }^{c, d}$, R. Borriss ${ }^{a, *}$ \\ a Institut für Biologie, Humboldt Universität Berlin, Chausseestrasse 117, D10115 Berlin, Germany \\ ${ }^{b}$ ABiTEP GmbH, Glienicker Weg 185, 12489 Berlin, Germany \\ c Bio-Protect GmbH, Lohnerhofstraße 7, 78467 Konstanz, Germany \\ d Universitaet Konstanz, LS Phytopathologie, Universitätsstrasse 10, D-78457 Konstanz, Germany
}

\section{A R T I C L E I N F O}

Article history:

Received 25 July 2008

Received in revised form

19 September 2008

Accepted 21 October 2008

\section{Keywords:}

Erwinia amylovora

Bacillus amyloliquefaciens

Fire blight control

Difficidin

Bacilysin

\begin{abstract}
A B S T R A T
Representatives of Bacillus amyloliquefaciens were shown to possess biocontrol activity against fire blight, a serious disease of orchard trees caused by Erwinia amylovora. Genome analysis of B. amyloliquefaciens FZB42 identified gene clusters responsible for synthesis of several polyketide compounds with antibacterial action. We show here that the antibacterial polyketides difficidin and to a minor extent bacillaene act efficiently against $E$. amylovora. Surprisingly, a mutant strain blocked in the production of difficidin (CH8 $\Delta d f n$ ) inhibited growth of $E$. amylovora and suppressed fire blight disease nearly in the same range as the wild type. In addition, a $s f p$ mutant $(\mathrm{CH} 3 \Delta s f p)$ unable to synthesize non-ribosomally lipopeptides and polyketides did still suppress growth of $E$. amylovora, suggesting that besides action of polyketides another antagonistic principle exist. A double mutant (RS06 $\Delta s f p \Delta b a c$ ) devoid in polyketide and bacilysin synthesis was unable to suppress growth of $E$. amylovora indicating that the additional inhibitory effect is due to production of bacilysin, a dipeptide whose synthesis does not depend on Sfp. We propose to use B. amyloliquefaciens strains with enhanced synthesis of difficidin and/or bacilysin for development of biocontrol agents efficient against fire blight disease.
\end{abstract}

\section{Introduction}

Fire blight caused by Erwinia amylovora is the most serious bacterial disease in apple and pear. During the last four decades it has spread throughout Europe. In 2007 heavy outbreaks of fire blight led to severe losses for apple growers in Germany, Austria and Switzerland. After being deposited on blossom surfaces by insects, E. amylovora multiplies on stigmas before it enters the plant tissue through nectarthodes of the blossoms or by wounds of leaves and twigs under warm and moist weather conditions. Since sanitation methods could not stop the spread of the disease, fire blight management by using appropriate biocontrol agents are a pressing need. Effective control can be achieved through application of streptomycin sulphate a method widely used in North America. However, the use of streptomycin has been banned by the European authorities, due to the risk of development of antibiotic resistance in non-target bacteria. Search of an environment friendly biological alternative is a permanent task of present research. Use of Aureobasidium pullulans ("BlossomProtect"). in organic fruit growing has been recommended (Kunz and Haug, 2006; Kunz et al., 2008).

\footnotetext{
* Corresponding author. Tel.: +49 30209381 37; fax: +49 $30209381: 27$

E-mail address: rainer.borriss@rz.hu-berlin.de (R. Borriss).
}

Unfortunately, Blossomprotect is not compatible in its application with fungicides used for apple scab control. Recently, two products based on Bacillus subtilis have been registered for fire blight control in Europe: Serenade ${ }^{\circledR}$, based on strain QST 713 and Biopro ${ }^{\circledR}$, based on strain BD 170 (Broggini et al., 2005). Spore-forming Bacillus based bioformulations offer advantages for biocontrol because of their long-term viability (Haas and Defago, 2005). A moderate efficiency of the two Bacillus based bioformulations has been established in several field trials in the USA and Germany (Aldwinckle et al., 2002; Laux et al., 2003; Kunz and Haug, 2006) suggesting a promising potential of Bacillus sp. in control of blossom infections by $E$. amylovora. However, results obtained with both bioproducts were found to be erratic, varying between $71 \%$ and $0 \%$ disease suppression. Therefore, we initiated this study to select effective Bacillus strains to develop an environmentally friendly, cost effective product with a high efficiency in fire blight control. Due to the limited capacity for field testing and the high costs of field trials, a fast but reliable two step screening procedure consisting of laboratory tests in vitro and an in vivo test system with detached apple blossoms (Kunz and Haug, 2006; Pusey, 1997) was applied.

Two compounds with strong antagonistic effect against $E$. amylovora, the polyketide difficidin and the dipeptide bacilysin were identified in this study. Both compounds were produced 
by Bacillus amyloliquefaciens FZB42, a representative of the group of plant-associated B. amyloliquefaciens strains, which proved to be especially efficient against fire blight in our screening procedure.

\section{Materials and methods}

\subsection{Bacterial strains used}

The indicator strains Erwinia amylovora Ea385 and Bacillus megaterium were provided by LfP Stuttgart and the Institute of Biology, Humboldt University, respectively.

116 bacterial strains of different origin were included in the first screening procedure. 77 Bacillus strains ("BS" and "MB") were kindly supplied by Dr. Bloemberg (Institute of Biology, Leiden University, The Netherlands). Other strains used in this study are from the laboratory stock of the chair of Phytopathology, University of Konstanz, Germany; ABiTEP GmbH, Berlin, Germany ("FZB"); and the Institute of Biology Humboldt University, Berlin, Germany. The Bacillus strain QST 713 isolated from the commercial product Serenade WPO (AgraQuest) was used for comparison.

\subsection{Mutant construction}

Construction of FZB42 mutant strains $\mathrm{CH} 3, \mathrm{CH} 8, \mathrm{CH} 14$ devoid in production of polyketides has been previously described (Chen et al., 2006). Mutants RS01 ( $\Delta b a c$ ) and RSO6 ( $\Delta b a c \Delta s f p$ ) devoid in bacilysin production were obtained in the following manner.

A PCR-fragment, involving bacA was amplified by the primer pair bcIFW 5'...ACAGAAGAGGAAAACGGACGGC, and bclRV $5^{\prime} \ldots$ ACGTCCAATCCGCCGAACTTG. The PCR-fragments were ligated into the vector PGEM-T to obtain the plasmid pbac12. The bacA gene was replaced by the $\mathrm{cm}^{\mathrm{R}}$ resistance cassette yielding plasmid pRS1. The linearized pRS1 was integrated into the genome of FZB42 by double cross-over recombination generating the knockout mutant RS1. Chromosomal DNA of mutant RS1 was integrated into the genome of the $\mathrm{CH} 3$ mutant by double cross-over recombination yielding mutant RSO6.

\subsection{Growth conditions}

Bacillus strains were grown in LB (Chen et al., 2006). Cultures for the production of polyketides were grown for $40 \mathrm{~h}$ at $30^{\circ} \mathrm{C}$ and $150 \mathrm{rpm}$ in Landy medium (Landy et al., 1948). For bacilysin production, the GA-medium was used. One litre GA-medium consisted of $\mathrm{K}_{2} \mathrm{HPO}_{4}, 7 \mathrm{~g}, \mathrm{KH}_{2} \mathrm{PO}_{4}, 2 \mathrm{~g}, \mathrm{MgSO}_{4} \cdot 7 \mathrm{H}_{2} \mathrm{O}, 0.1 \mathrm{~g},\left(\mathrm{NH}_{4}\right) \mathrm{SO}_{4}$, $1 \mathrm{~g}$, yeast extract, $0,05 \mathrm{~g}$, $\mathrm{Fe}\left(\mathrm{SO}_{4}\right)_{3} \cdot 6 \mathrm{H}_{2} \mathrm{O}, 0.15 \mathrm{mg}, \mathrm{MnSO}_{4} \cdot \mathrm{H}_{2} \mathrm{O}, 5 \mathrm{mg}$, $\mathrm{CuSO}_{4} \cdot 5 \mathrm{H}_{2} \mathrm{O}, 0.16 \mathrm{mg}$ and sucrose, $13 \mathrm{~g}$. Colonies of $\mathrm{FZB} 42$ and the mutant strains were inoculated in $10 \mathrm{ml} \mathrm{GA}$-medium. The cultures were grown at $29^{\circ} \mathrm{C}$ and $165 \mathrm{rpm}$ for $24 \mathrm{~h}$. The indicator strains $B$. megaterium and $E$. amylovora were grown in Luria broth (LB), if not otherwise stated.

\subsection{Dual culture}

Bacillus strains were grown overnight in NBS medium (Sifin, Berlin-Weissensee, Germany) consisting of pancreatic peptone (casein, meat) $2.25 \%$, glucose $0.1 \%, \mathrm{KH}_{2} \mathrm{PO} 40.2 \%, \mathrm{~K}_{2} \mathrm{HPO} 40.2 \%, \mathrm{NaCl}$ $0.3 \%$ at $27^{\circ} \mathrm{C}$. Ea385 was grown for $48 \mathrm{~h}$ on $2 \%$ NBS agar. Before inoculating the mixed-species culture, Ea385 was diluted to a concentration of $1 \times 10^{7}$ cells $/ \mathrm{ml}$ and the Bacillus strain was adjusted to a concentration of $5 \times 10^{7}$ cells $/ \mathrm{ml}$. After $24 \mathrm{~h}$ growth, concentration of Ea385 in the dual culture was determined by serial dilutions plated onto McConkey agar.

\subsection{Purification of polyketides by XAD16}

For preparation of samples used for HPLC and bioautography, $7 \mathrm{ml}$ culture volume of each Bacillus strain were centrifuged for $10-40 \mathrm{~min}$ at $11,000 \mathrm{rpm}$ and purified on an amberlite XAD16 column $(6 \mathrm{~g})$ by washing with $400 \mathrm{ml}$ distilled water and eluting by $14 \mathrm{ml} \mathrm{100 \%} \mathrm{methanol.} \mathrm{Eluates} \mathrm{were} \mathrm{dried} \mathrm{in} \mathrm{a} \mathrm{rotation} \mathrm{evaporator}$ and resolved in $550 \mu \mathrm{l}$ methanol containing $10 \%$ distilled water.

\subsection{Bioautography and agar diffusion test}

When cultures of the indicator strains B. megaterium and $E$. amylovora reached an $\mathrm{OD}_{600 \mathrm{~nm}}$ of 1.0 culture was diluted $1: 40$ in appropriate medium solidified with $1 \%$ agar. $20 \mu \mathrm{l}$ of the Bacillus and $60 \mu \mathrm{l}$ of $E$. amylovora supernatant were used in agar diffusion test.

Bioautography of polyketides was performed essentially as previously described (Chen et al., 2006). Samples were purified with XAD16 resin. $10 \mu \mathrm{l}$ of the samples purified by XAD16 as described above were loaded onto thin-layer chromatography (TLC) aluminum sheets for $B$. megaterium bioautography; whilst $30 \mu \mathrm{l}$ was necessary for the $E$. amylovora test system. TLC was performed with chloroform-methanol-water at $65: 25: 4$, vol/vol. For bacilysin detection $30 \mu \mathrm{l}$ supernatant of the culture grown in GA medium were spotted onto TLC sheets. Mobile phase consisted of 1-butanol/acetic acid/water $4: 1: 1(\mathrm{v} / \mathrm{v} / \mathrm{v})$.

\subsection{Assay of polyketides and bacilysin}

High pressure liquid chromatography was performed with the HPLC 1200 device from Agilent, Waldbronn, Germany, essentially as previously described (Schneider et al., 2007). In brief, $10 \mu \mathrm{l} \mathrm{sam-}$ ple was injected onto a HPLC column (ZoRBX Eclipse XDB-C18, $4.6 \times 150 \mathrm{~mm}, 5 \mu \mathrm{m}$, Agilent). The temperature was kept at 30 " $\mathrm{C}$ during the experiment. The run was performed with a flow rate of $1.5 \mathrm{ml} / \mathrm{min}$ and a gradient of solvents $\mathrm{A}(0.1 \%, \mathrm{v} / \mathrm{v} \mathrm{HCOOH})$ and $\mathrm{B}$ $\left(\mathrm{CH}_{3} \mathrm{CN}\right)$, which reached $100 \% \mathrm{~B}$ after $10 \mathrm{~min}$. Concentration of $100 \%$ $\mathrm{CH}_{3} \mathrm{CN}-\mathrm{HCOOH}$ was held for further two min. To equilibrate the column, it was treated with $5 \% \mathrm{CH}_{3} \mathrm{CN}-\mathrm{HCOOH}$ for $3 \mathrm{~min}$. A diode-array detector in range from $190 \mathrm{~nm}$ to $550 \mathrm{~nm}$ was used for detecting peaks. All the polyketides were detected at $280 \mathrm{~nm}$; bacilysin was detected at $230 \mathrm{~nm}$. For quantitative assays, the area of polyketide peaks were calculated with the "auto integrate" software of Agilent.

\subsection{Efficiency of test strains against E. amylovora on detached blossoms}

An in vivo test-system with detached apple blossoms was established according to Pusey (1997). Apple trees ('Gala') were stored at $2^{\circ} \mathrm{C}$ in the dark from January to August. Every week a group of trees was transferred to the greenhouse to force them to bloom. The blossoms were cut and maintained with cut peduncle submerged in $10 \%$ sucrose in plastic racks $\left(23^{\circ} \mathrm{C}, 100 \% \mathrm{rH}\right)$. Blossoms were sprayed with a suspension of Ea385 $\left(10^{6} \mathrm{cfu} / \mathrm{ml}\right)$ in water until runoff. Test preparations of Bacillus strains were sprayed to the blossoms $1 \mathrm{~h}$ after inoculation. The number of blossoms with bacterial ooze at the peduncle was counted 6 days after the inoculation (Fig. 1). The experiments were repeated three to four times unless otherwise stated.

\section{Results and discussion}

\subsection{Screening of Bácillus strains}

Initially, $\mathbf{1 1 6}$ strains were tested in competitive mixed culture experiments (see Section 2) for their ability to suppress growth of $E$. 


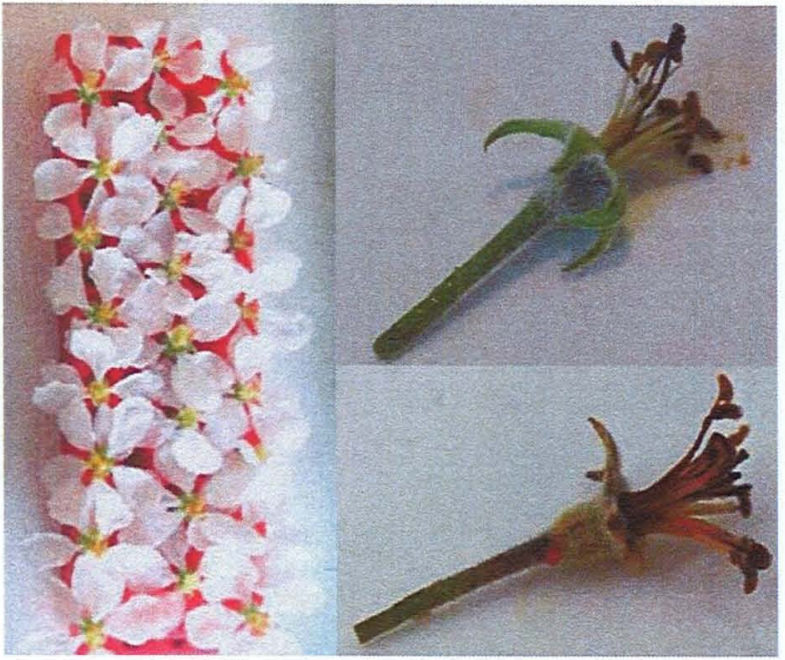

Fig. 1. Left: Detached apple blossoms in racks prior to inoculation and treatment Right: Blossom after 6 days of incubation at $20^{\circ} \mathrm{C}$ and $100 \%$ relative humidity. Top: healthy blossom. Below: Blossom with black peduncle and bacterial ooze due to infection by $E$. amylovora.

amylovora (Supplementary material: Table 1). The efficiency of the strains depended on the incubation temperature. In general, strains showed higher efficiency at $27^{\circ} \mathrm{C}$ than at $20^{\circ} \mathrm{C}$. During apple bloom in mid-Europe, temperatures of $27^{\circ} \mathrm{C}$ are rarely reached. Therefore, the suppression of $E$. amylovora at $20^{\circ} \mathrm{C}$ is a prerequisite for high activity in the field. Strain QST713, the active constituent of the biocontrol agent "Serenade ${ }^{\circledR}$ " was used for comparison. Around 20 strains including QST713 and FZB42 suppressed E. amylovora growth in mixed culture completely (Table 1). Those strains were selected for further experiments with detached blossoms.

On detached blossoms the recommended concentration of the formulated product Serenade WPO was less effective than the undiluted culture filtrate of QST713. Interestingly, six strains (FZB 45, FZB42, FZB13, FDK33, FDK21 and KF60) all belonging to B. amyloliquefaciens, displayed a higher efficiency than QST713 (Tab. 1).
We have shown previously that QST713, formerly misclassified as B. subtilis, is also a representative of the group of plant-associated B. amyloliquefaciens strains, which are occurring inside the plant rhizosphere (Chen et al., 2007).

\subsection{Quantitative HPLC analysis of polyketide production}

FZB42 and other plant-associated B. amyloliquefaciens strains are known for their production of antibacterial polyketides. Polyketide megasynthases responsible for synthesis of bacillaene, macrolactin and difficidin are encoded by three giant gene clusters that are located at different sites of the FZB42 genome (Chen et al., 2007). Those polyketides, especially difficidin, have been shown to act against Gram-positive and Gram-negative bacteria, including the plant pathogen Erwinia carotovora (Chen et al., 2006) and might be good candidates for being the "antagonistic principle" acting against the causative agent of fire blight. A previous screening for presence of those polyketides in bacilli revealed that occurrence of all those three antibiotics is limited to the group of plant-associated B. amyloliquefaciens. Notably, difficidin, the most efficient antibacterial compound in FZB42, is restricted in its occurrence to that selected group of bacteria (Schneider et al., 2007).

For identification and a quantitative analysis of polyketides by high pressure liquid chromatography (HPLC), we took advantage that distinct peaks obtained after the HPLC run can be distinguished. Using mutant strains deficient in production of difficidin (CH8), macrolactin, and bacillaene ( $\mathrm{CH} 14$ ) or completely devoid in non-ribosomal synthesis of lipopeptides and polyketides (CH3) and supported by the availability of the specific UV-absorption maxima of those compounds we were able to assign the profile generated by HPLC to distinct polyketides (see Fig. 2). The quantities corresponding to the isoforms were calculated as described in Section 2.

Our analysis revealed that nearly all the strains that have proven as especially efficient in the blossom test and in suppressing $E$. amylovora, did produce the spectrum of the three polyketides, which are unique for the $B$. amyloliquefaciens group. Whilst the main polyketide produced by B. amyloliquefaciens FZB42 and QST713 is difficidin, other strains with high polyketide production exhib-

Table 1

Efficiency (\%) of the test strains in suppressing Erwinia amylovora in dual culture and on detached blossoms.

\begin{tabular}{|c|c|c|c|c|c|c|}
\hline \multirow[t]{2}{*}{ Strain } & \multicolumn{2}{|c|}{ Dual culture } & \multicolumn{2}{|c|}{ Blossom test } & \multirow[t]{2}{*}{ Species } & \multirow[t]{2}{*}{ Source } \\
\hline & $20^{\circ} \mathrm{C}$ & $277^{\circ} \mathrm{C}$ & $100 \%$ & $20 \%$ & & \\
\hline FZB 45 & 100 & 100 & 98 & n.d. & B. amyloliquefaciens & ABiTEP GmbH \\
\hline FZB 42 & 100 & 100 & 98 & $42^{b}$ & B. amyloliquefaciens & ABiTEP GmbH \\
\hline FZB 13 & 100 & 100 & 96 & $95^{b}$ & B. amyloliquefaciens & ABiTEP GmbH \\
\hline FDK 33 & 100 & 100 & 96 & $95^{\mathrm{b}}$ & B. amyloliquefaciens & University of Konstanz \\
\hline FDK 21 & 98 & 100 & 96 & $83^{b}$ & B. amyloliquefaciens & University of Konstanz \\
\hline KF 60 & 99 & 99 & 87 & $83^{b}$ & B. amyloliquefaciens & University of Konstanz \\
\hline QST713 & 100 & 100 & 76 & n.d.c & B. amyloliquefaciens & Serenade WPO (AgraQuest) \\
\hline FZB 108 & 100 & 100 & 66 & n.d. & B. subtilis & ABITEP GmbH \\
\hline MB 2 & 100 & 100 & n.d. & 58 & Bacillus mojavensis & Leiden University \\
\hline FZB 24 & 100 & 100 & 65 & $47^{b}$ & B. amyloliquefaciens & ABiTEP GmbH \\
\hline FZB 109 & 99 & 100 & 57 & n.d. & B. amyloliquefaciens & ABiTEP GmbH \\
\hline FDK 24 & 100 & 100 & 39 & n.d. & B. velezensis & University of Konstanz \\
\hline BS 19 & 100 & 100 & n.d. & 22 & Bacillus spp. & Leiden University \\
\hline MB 8 & 100 & 100 & n.d. & 21 & Bacillus spp. & Leiden University \\
\hline MB 33 & 100 & 99 & n.d. & 13 & Bacillus spp. & Leiden University \\
\hline MB 31 & 100 & 100 & n.d. & 11 & Bacillus spp. & Leiden University \\
\hline MB 23 & 100 & 100 & n.d. & 10 & Bacillus spp. & Leiden University \\
\hline MB 27 & 100 & 100 & n.d. & 6 & Bacillus spp. & Leiden University \\
\hline BS 18 & 100 & 100 & n.d. & 3 & Bacillus spp. & Leiden University \\
\hline MB 25 & 100 & 100 & n.d. & 1 & Bacillus spp. & Leiden University \\
\hline
\end{tabular}

a Dual cultures consisting of Erwinia amylovora and the test strain were incubated at temperatures of either $20^{\circ} \mathrm{C}$ or $27^{\circ} \mathrm{C}$. The efficiency on blossoms was tested by spraying undiluted (100\%) and water diluted (20\%) culture broths.

b The experiment was done only once.

c n.d., not determined. 

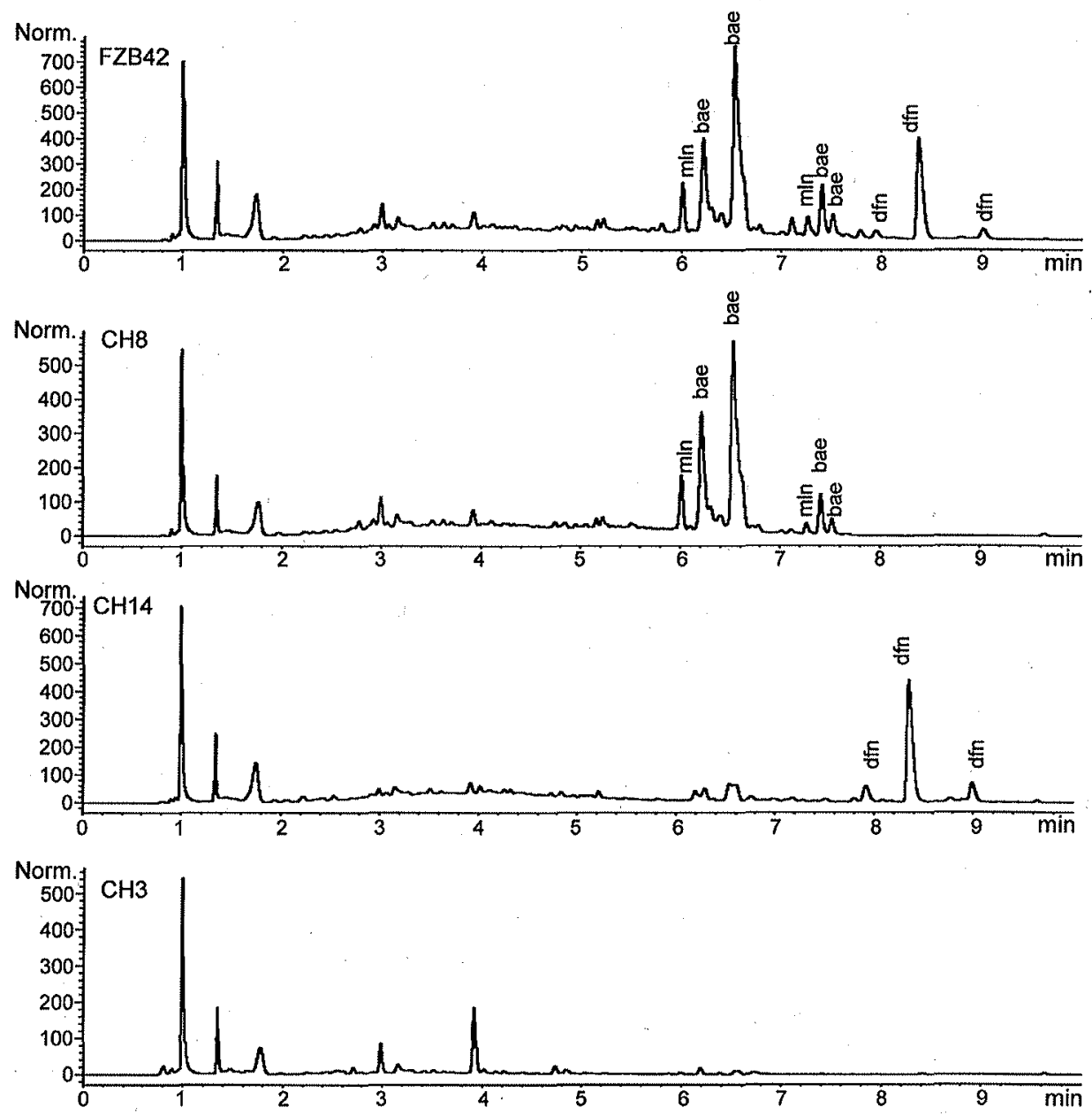

Fig. 2. HPLC analysis and identification of single metabolites in culture supernatants of FZB42 and the mutant strains $\mathrm{CH} 3(\Delta s f p)$, $\mathrm{CH} 8(\Delta d f n)$ and $\mathrm{CH} 14(\Delta b a e ~ \Delta m I n)$ grown in Landy medium. mln, macrolactin; dfn, difficidin; bae, bacillaene.

Table 2

Polyketide profiles and efficiency against E. amylovora determined in dual culture (1) and in detached blossom (2) .

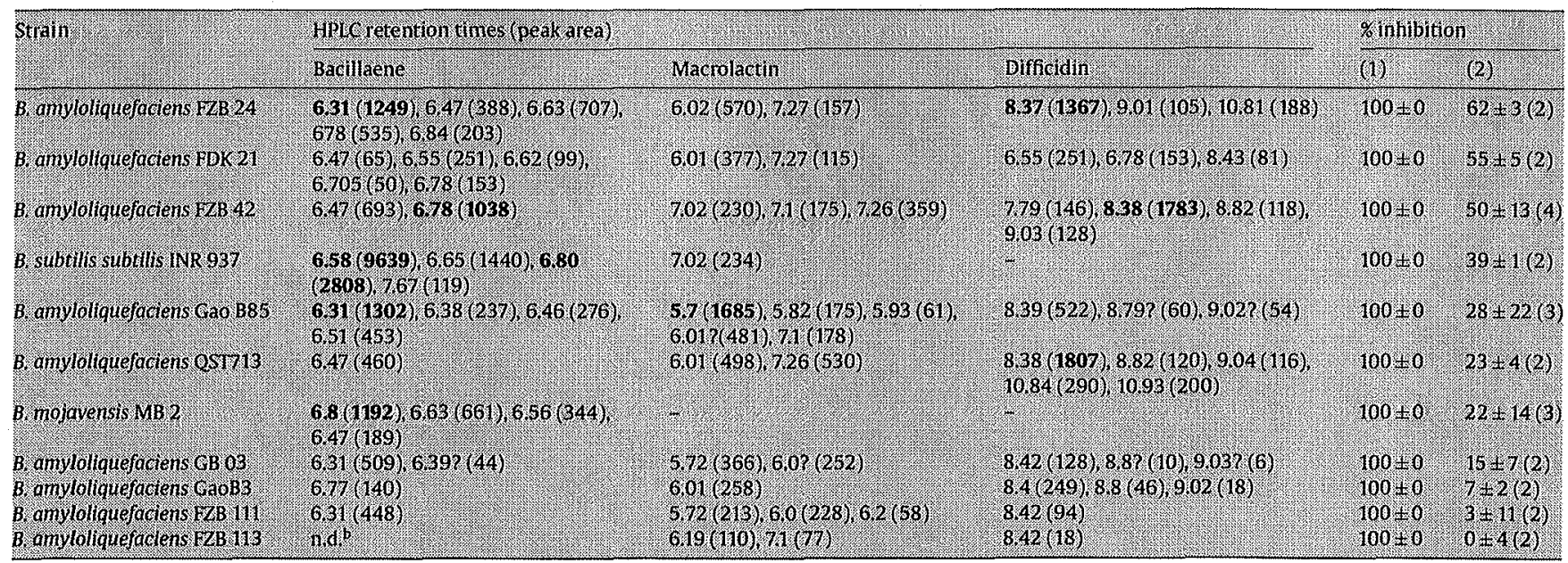

a Prominent peaks of the polyketides are indicated in bold letters. For the blossom test Bacillus strains were grown in an optimised medium. Water diluted culture broth (1\%) was directly sprayed on the detached apple blossoms. Numbers in brackets indicate number of repetitions.

b n.d., not detected. 
Table 3

Effect of B. amyloliquefaciens FZB42 and its mutant strains $\mathrm{CH} 8$ and $\mathrm{CH} 14$ on detached blossoms infected with Erwinia amylovora ${ }^{\mathrm{a}}$.

\begin{tabular}{|c|c|c|c|c|c|}
\hline \multirow[t]{2}{*}{ Strain } & \multirow[t]{2}{*}{ Geno-type } & \multicolumn{3}{|c|}{ Synthesis of polyketides } & \multirow[t]{2}{*}{ Efficiency [\%] on detached blossoms } \\
\hline & & Bacillaene & Macrolactin & Difficidin & \\
\hline FZB 42 & Wild-type & Yes & Yes & Yes & $84 \pm 7(3)$ \\
\hline $\mathrm{CH} 8$ & $\triangle d f n:: e r m A M$ & Yes & Yes & No & $79 \pm 5(3)$ \\
\hline $\mathrm{CH} 14$ & $\Delta$ bae::cat $\Delta$ min::neo & No & No & Yes & $89 \pm 12(3)$ \\
\hline
\end{tabular}

a The number in brackets indicate number of repetitions.

ited a nearly equal ratio between difficidin and bacillaene (FZB24) or a production of either bacillaene and macrolactin was more pronounced (GaoB85, Table 2). A remarkably high production of bacillaene was registered in B. subtilis INR937, a strain also proven as being efficient in suppression of $E$. amylovora. However, the best results in suppressing the fire blight bacterium in vitro (mixed culture) and in vivo (detached blossom) were obtained with the $B$. amyloliquefaciens strains FZB24, FZB42 and FDK21 (Table 2).

\subsection{The polyketide difficidin produced by FZB42 suppresses $E$ amylovora}

To ascertain the role of difficidin and other polyketides in suppressing fire blight by $B$. amyloliquefaciens, we compared the effect exerted on $E$. amylovora infected blossoms by FZB 42 with that of polyketide deficient mutants generated by targeted mutagenesis of competent FZB42 cells via homologous recombination (Chen et al., 2006). As expected, the suppressive effect exerted by $\mathrm{CH} 14$ the mutant strain producing only difficidin but no macrolactin and bacillaene, was found equal or slightly higher, than in FZB42 wild-strain underlining importance of difficidin. However, strain $\mathrm{CH} 8$, deficient in difficidin production was only slightly reduced in its ability to suppress fire blight symptoms on detached blossoms (Table 3).

The following mutant strains were used to investigate in vitro suppressing effects of difficidin and bacillaene on $E$. amylovora by bioautography (see Section 2): CH8 ( $\Delta d f n$ ), deficient in difficidin but unaffected in bacillaene and macrolactin production, $\mathrm{CH} 14$ ( $\Delta$ bae $\Delta m l n$ ), deficient in production of macrolactin and bacillaene but proficient in difficidin formation and $\mathrm{CH} 3(\Delta s f p)$, unable to produce any polyketide or lipopeptide due to a deletion introduced into the gene encoding 4'-phosphopantetheinyl transferase, SfP, an enzyme catalysing a necessary processing step in non-ribosomal synthesis of lipopeptides and polyketides. All strains were grown in Landy medium, a medium specifically designed to facilitate production of lipopeptides and polyketides. Bioautography showed that among the compounds present in the supernatant of FZB42, difficidin strongly inhibited growth of the sensitive $B$. megaterium indicator strain, whilst bacillaene and the lipopeptides surfactin had a minor effect. Growth inhibition caused by difficidin, the only polyketide produced by mutant $\mathrm{CH} 14$, was in the same range as in the wild-type. Slight growth repression due to presence of bacillaene and surfactin in mutant $\mathrm{CH} 8$ was also registered. However, strain $\mathrm{CH} 3$, was completely unable to produce any compound that suppress growth of the B. megaterium indicator strain (Fig. 3A). Similar effects were observed when $E$. amylovora was used as indicator strain in bioautography. Only growth inhibition caused by difficidin and to a smaller extent by bacillaene was detectable (Fig. 3B). However, agar diffusion tests performed with the cell-free supernatants of FZB42 and the three mutant strains mentioned above yielded a different scenario. As expected, growth of $B$. megaterium was clearly inhibited by supernatants of FZB42 and the difficidin producer $\mathrm{CH} 14$, whilst the inhibiting effect exerted by $\mathrm{CH} 8$ (devoid in difficidin) was clearly reduced and $\mathrm{CH} 3$ did not affect growth of B. megaterium at all (Fig. 3C). When E. amylovora was used as indicator, growth inhibiting effect exerted by $\mathrm{CH} 8$ still remained low underlining the main antibacterial action of difficidin. But to our surprise, the inhibiting effect exerted by $\mathrm{CH} 3$ on $E$. amylovora was
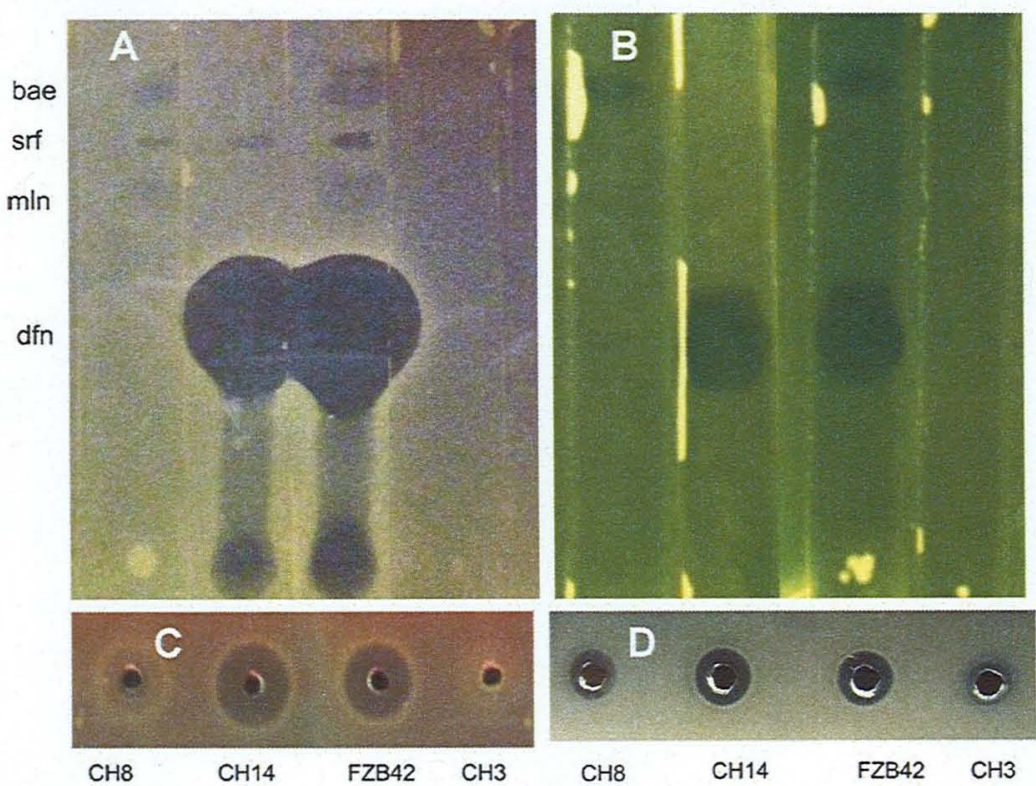

Fig. 3. Bioautography and agar diffusion test of $\mathrm{FZB} 42$ and mutant strains $\mathrm{CH} 3, \mathrm{CH} 8$ and $\mathrm{CH} 14$ grown in Landy medium. 3A: Bioautography with indicator strain B. megaterium; 3B: Bioautography with indicator strain E. amylovora; 3C: Agar diffusion test with indicator strain B. megaterium; 3D: Agar diffusion test with indicator strain E. amylovora. For details see text. 
comparable with that of FZB42 and CH14, as well, although no Sfpdependent synthesis of polyketides and lipopeptides did occur in that strain (Fig. 3D).

\subsection{The dipeptide bacilysin acts as an additional suppressor of the growth of E. amylovora}

To identify the molecular reason for the observed suppression of E. amylovora by $\mathrm{CH} 3$, we searched in the FZB42 genome for gene clusters involved in Sfp-independent synthesis of secondary metabolites. Only one gene cluster involved in biosynthesis of the dipeptide bacilysin and its antibiotic constituent anticapsin was identified (Chen et al., 2007). The gene cluster, detected in FZB42 at 3,593,877-3,599,784, displayed the same gene organisation as the one known for $B$. subtilis 168 , in which the genes bacABCDE and $y w f G$ have been shown necessary for bacilysin synthesis (Steinborn et al., 2005). Similarity between both gene clusters was around $84-93 \%$ identity on amino acid level. The bacilysin molecule contains an L-alanine residue at the N-terminus and a non-proteinogenic amino acid, L-anticapsin, at the C-terminus (Walker and Abraham, 1970). Antibiotic action is achieved by the anticapsin moiety, which becomes released after uptake in susceptible cells and blocks glucosamine synthetase, an essential enzyme of cell wall biosynthesis (Kenig et al., 1976). Products of the bacDE genes function in non-ribosomal but Sfp independent amino acid ligation and immunity, respectively (Steinborn et al., 2005).

A double mutant, RSO6 ( $\Delta s f p, \Delta b a c$ ), simultaneously blocked in non-ribosomal synthesis of lipopeptides and polyketides and in

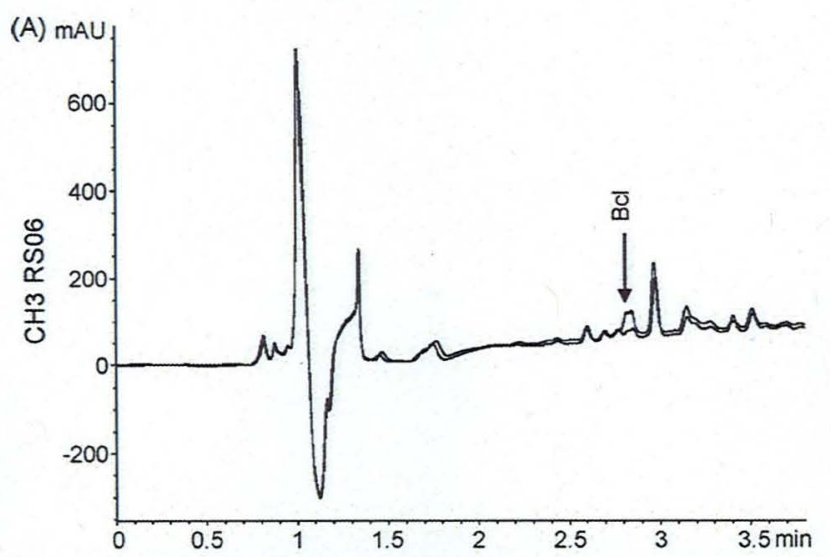

(B)

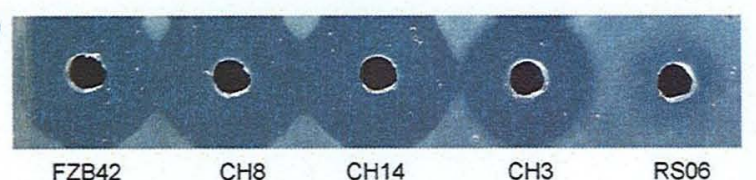

(C)

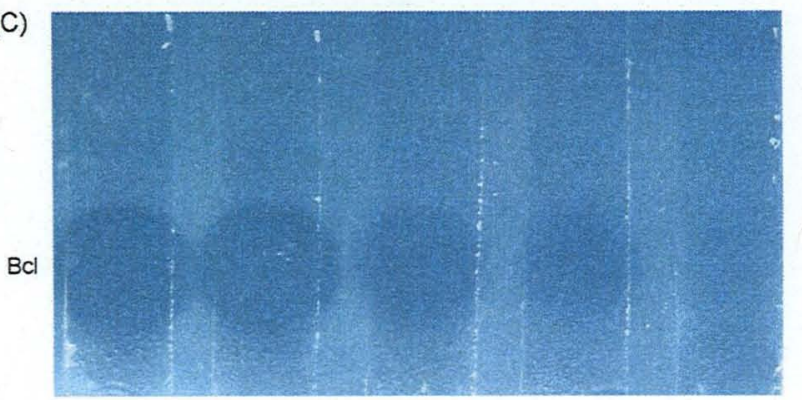

Fig. 4. HPLC analysis (A), agar diffusion test (B) and bioautography (C) with FZB42 and the mutant strains $\mathrm{CH} 3(\Delta s f p), \mathrm{CH} 8(\Delta d f n), \mathrm{CH} 14(\Delta b a e \Delta m l n)$ and $\mathrm{RSO6}(\Delta s f p$ $\triangle b a c)$ grown in GA medium. For details see text. Bcl, bacilysin. production of bacilysin (Fig. 4A) was used to investigate repressing effect of bacilysin on growth of E. amylovora. Strains FZB42, $\mathrm{CH} 8, \mathrm{CH} 14, \mathrm{CH} 3$, and RS06 were grown in GA medium specifically designed for bacilysin production (see Section 2). Bioautography (Fig. 4C) revealed that bacilysin produced by all that strains under those conditions, except RSO6, inhibits growth of $E$. amylovora. Agar diffusion tests performed with the supernatants of FZB42, CH8 and $\mathrm{CH} 14$ yielded strong inhibition zones onto the $E$. amylovora indicator lawn. The zone of inhibition caused by supernatant of $\mathrm{CH} 3$ was also in a comparable size. On the contrary, the double mutant RS06 did not significantly repress growth of $E$. amylovora, suggesting that bacilysin acts as an important antagonist of E. amylovora (Fig. 4B).

\section{Conclusion}

Plant-associated B. amyloliquefaciens, e.g. FZB42, are efficient in suppressing plant pathogenic bacteria, including E. amylovora, the causative agent of fire blight disease in orchard trees. Trials performed with inoculated detached blossoms and field trials (Fried, 2007) have corroborated the results of the in vitro experiments reported here. Bacillus based bioformulations have important advantages in fire blight control compared to other biocontrol agents:

1. Bacillus endospores with long-term viability guarantee constant product quality over a long period of time.

2. Together with the active substances, living spores can be applied to blossoms of apple or pear trees. Spores might be able to germinate and multiply there and could be carried to other blossoms by insects (Broggini et al., 2005). Given that FZB42 can produce difficidin and bacilysin during colonising the blossoms, continuous supply of antagonistic compounds, directly at the site where E. amylovora normally occurs, would be possible.

3. In contrast to other biocontrol agents used in fire blight control, the active principles suppressing the devasting disease of apple and pear trees have been identified as being difficidin, one of the three polyketides produced by FZB42, and the antimicrobial dipeptide bacilysin.

The identification of these two compounds as efficient suppressors of E. amylovora will facilitate future directed development of optimised Bacillus based biocontrol agents as alternatives to streptomycin sulphate and other toxic chemo-pesticides which become increasingly restricted in their application.

\section{Acknowledgements}

The work was supported by a research grant from the German Ministry of Education and Research (BMBF; 0313805U GenoMikPlus) to R.B., S.K. and H.J. and partly funded by the 6th Framework of the European Commission (Bactofruct; Project No. 512622) with grants to H.J. and S.K. Initial strain survey has been performed by Dr. Daljeet Singh during his research stay at the University of Konstanz. We are also grateful to Prof. Dr. G. Bloemberg, and to Prof. Dr. K. Mendgen for providing Bacillus strains.

\section{References}

Aldwinckle, H.S., Bhaskara Reddy, M.V., Norelli, L., 2002. Evaluation of fire blight infection of apple blossoms and shoots with SAR inducers, biological agents, a growth regulator, copper compound, and other materials. Acta Horticult. 590 , 325-331.

Broggini, G.A.L., Duffy, B., Holliger, E., Schärer, H.-J., Gessler, C., Patocchi, A., 2005. Detection of the fire blight biocontrol agent Bacillus subtilis BD170 (Biopro $\left.{ }^{\circledR}\right)$ in a Swiss apple orchard. Eur. J. Plant Pathol. 111,93-100.

Chen, X.H., Vater, J., Piel, J., Franke, P., Scholz, R., Schneider, K., Koumoutsi, A., Hitzeroth, G., Grammel, N., Strittmatter, A.W., Gottschalk, G., Süssmuth, R.D., 
Borriss, R., 2006. Structural and functional characterization of three polyketide synthase gene clusters in Bacillus amyloliquefaciens FZB42. J. Bacteriol. 188, $4024-4036$.

Chen, X.H., Koumoutsi, A., Scholz, R, Eisenreich, A., Schneider, K., Heinemeyer, I.,

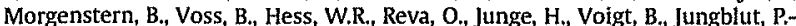
J., Vater, J., Süssmuth, R., Liesegang, H., Strittmatter, A., Gottschalk, G., Borriss,
R., 2007. Comparative analysis of the complete genome sequence of the plant growth-promoting bacterium Bacillus amyloliquefaciens FZB42. Nat. Biotechnol. $25,1007-1014$.

Fried, A, 2007. Feuerbrand-Bekämpfungsversuch und Verträglichkeitsprüfung von Feuerbrandpräparaten 2007. Obstbau. 33, 72-75.

Haas, D., Defago, G., 2005. Biological control of soil-borne pathogens by fluorescent pseudomonads. Nat. Rev. Microbiol. 3, 307-319.

Kenig, M., Vandamme, E., Abraham, E.P., 1976. The mode of action of bacilysin and anticapsin and biochemical properties of bacilysin resistant mutants. J. Gen. Microbiol. 94, 46-54

Kunz, S., Haug, P., 2006. Development of a strategy for fire blight control in organic fruit growing. In: FÖKO, e.V. (Ed.), 12th International Conference on Cultivation Technique and Phytopathological Problems in Organic Fruit-Growing. FOKKO e.V., Weinsberg, pp. 145-150.
Kunz, S., Schmitt, A., Haug, P., 2008. Field testing of strategies for fire blight control in organic fruit growing. In: FÖKO, e.V.(Ed.), 13th International Conference on Cultivation Technique and Phytopathological Problems in Organic Fruit-Growing. FÖKO e.V., Weinsberg, pp. 299-305.

Landy, M. Warren, G.H. Roseman, S.B. Colio, L.G., 1948. Bacillomycin, an antibiotic from Bacillus subtilis active against pathogenic fungi. Proc. Soc. Exp. Biol. Med. $67,539-541$.

Laux, P., Wesche, J., Zeller, W., 2003. Field experiments on biological control of fire blight by bacterial antagonists. J. Plant Disease Prot. 110, 401-407.

Pusey, P.L., 1997. Crab apple blossoms as a model for research on biological control of fire blight. Phytopathology 87, 1096-1102.

Schneider, K., Chen, X.H., Vater, J., Franke, P., Nicholson, G., Borriss, R., Süssmuth, R.D, 2007. Macrolactin is the polyketide biosynthesis product of the pks2 cluster of Bacillus amyloliquefaciens FZB42. J. Nat. Prod. 70, 1417-1423.

Steinborn, G., Hajirezaei, M.-R., Hofemeister, J., 2005, bac genes for recombinan bacilysin and anticapsin production in Bacillus host strains. Arch. Microbiol. 183 $71-79$.

Walker, J.E Abraham, E.P, 1970. The structure of bacilysin and other products of Bacillus subtilis, Biochem. J. 118, 563-570. 